Article

\title{
Strategic Maintenance Scheduling of an Offshore Wind Farm in a Deregulated Power System
}

\author{
Peyman Mazidi ${ }^{1,2, *}$, Yaser Tohidi ${ }^{2}$ and Miguel A. Sanz-Bobi ${ }^{1}$ \\ 1 Institute for Research in Technology (IIT), Comillas Pontifical University, 28015 Madrid, Spain; \\ masanz@comillas.edu \\ 2 Electric Power and Energy Systems Department, KTH Royal Institute of Technology, \\ SE-10044 Stockholm, Sweden; tohi@kth.se \\ * Correspondence: peyman@comillas.edu or mazidi@kth.se; Tel.: +34-91-542-2800 (ext. 2749)
}

Academic Editors: Pierluigi Siano and Miadreza Shafie-khah

Received: 21 December 2016; Accepted: 1 March 2017; Published: 6 March 2017

\begin{abstract}
This paper proposes a model for strategic maintenance scheduling of offshore wind farms (SMSOWF) in a deregulated power system. The objective of the model is to plan the maintenance schedules in a way to maximize the profit of the offshore wind farm. In addition, some network constraints, such as transmission lines capacity, and wind farm constraints, such as labor working shift, wave height limit and wake effect, as well as unexpected outages, are included in deterministic and stochastic studies. Moreover, the proposed model provides the ability to incorporate information from condition monitoring systems. SMSOWF is formulated through a bi-level formulation and then transformed into a single-level through Karush-Kuhn-Tucker conditions. The model is validated through a test system, and the results demonstrate applicability, advantages and challenges of harnessing the full potential of the model.
\end{abstract}

Keywords: bi-level optimization; maintenance scheduling; deregulated power system; offshore wind farm

\section{Introduction}

Environmental concerns accompanied by exhaustion of fossil fuels have interest in the integration of renewable energy sources (RES). Among various types of RES, wind energy in the form of wind farms has evolved rapidly and is replacing conventional energy sources in several power systems. The estimates for offshore wind show large and rapid growth where offshore is expected to produce about $10 \%$ of the global installed capacity by 2020 [1] from the first offshore wind farm (OWF) at Vindeby, Denmark in 1991. Operation and maintenance (O\&M) costs of an OWF are high, and O\&M costs can reach up to $30 \%$ of the total cost [2,3], where they have reached up to $23 \%$ in some European offshore farms [4]. Moreover, if the farm is located at remote offshore areas, its availability can reduce down to 70\%, as the vessel accessibility decreases [5]. In addition, as future OWFs will be further away from the coast (e.g., $200 \mathrm{~km}$ ), arranging the transportation vessel to the farm becomes very important, as it could take more than $10 \mathrm{~h}$ to reach a site [6-8].

The downtime due to maintenance can cause loss of profit. Especially, in the deregulated power system where the electricity prices vary constantly, finding the optimum maintenance window is a difficult task. There are several models focused on preventive maintenance scheduling of components in a wind turbine (WT) through failure rate and age modeling [9-12]. Apart from the uncertainty in input data, the uncertainty in failure rate, age or degradation models and the corresponding threshold values are the most important parameters that influence the performance of such models [13-15].

Preventive maintenance scheduling of OWFs can be divided into two categories of passive and active based on their relation with the market. Passive maintenance scheduling denotes that the 
schedules of maintenance are obtained with the objective of minimizing the operation cost of the power system without considering opportunities of making profit, whereas active maintenance scheduling stands for maintenance decision making with the objective of maximizing the profit resulting from the consideration of varying electricity prices. Furthermore, with the developments in the condition monitoring systems in the wind industry and the requirement for quick reactions towards alarm and failure signals, in deregulated power systems, active maintenance scheduling carries more importance and holds greater interests for the wind farm operators.

A recent review study [16] presents currently applied maintenance logistics organization for OWFs and concludes that operational maintenance should try to better adapt to the dynamics of the issues. The study in [17] analyzes simulation models and suggests that future studies should try to cover optimization perspectives. Several models with a focus on O\&M strategies are reviewed in [18]. Studies [19-21] investigate the impact of meteorological parameters on availability and maintenance planning where, for instance, significant changes in production loss and availability are observed, and the addition of alternative access methods, e.g., a helicopter, is suggested for future studies. Research works [22,23] perform cost-based optimizations by considering different transport vessels, seasonal environment, component changes, etc., in order to find the optimum preventive maintenance schedules and the number of permanent maintenance teams. In addition, a sensitivity analysis is carried out that deduces that the electricity price is one of the major parameters affecting the outcomes. Moreover, $[24,25]$ mention that there is also a need for improving the way the condition monitoring features are being currently exploited as the transportation costs and scheduling of the transport mediums include the majority of the costs [26]. Furthermore, the study in [27] performs a sensitivity analysis in order to find out the most important parameters affecting O\&M. It concludes that repair duration and labor working shift length are two of the most significant variables influencing the cost; maintenance resource availability and components' failure rates are the other two impacting availability.

There are several studies addressing active preventive maintenance scheduling of wind farms in a network comprised of multiple types of power plants [28-31]. Research work [28] performs maintenance scheduling where particular wind power plant-related matters (e.g., varying wind) are neglected. The study [30] solves the maintenance scheduling while neglecting the strategic behavior of OWF operator. A maintenance scheduling is performed in [29] by maximizing the reliability of a power system with wind generation where two wind particular constraints of wind variations and weather conditions are used. The recent work [31] reviews maintenance scheduling studies in the electricity industry and provides several propositions, such as modifying the models based on the regulated and deregulated systems, addressing price volatility, tailoring the objectives, which enhances strategic behavioral analysis in this area, and the addition of specific constraints for different types of power plants. For instance, currently, when a power system comprised of multiple power plant types is considered, maintenance schedules of wind power plants (regardless of their constraints) are handled similar to other power plants with one single maintenance schedule for the whole farm. This includes many simplifications that distances it from an actual case. For example, the impact of the wake effect or vessel availability is ignored. Indeed, this assumption is not realistic as each WT can have a different maintenance time. Therefore, there is lack of a study that performs active maintenance scheduling with being detailed on the wind farm and turbines in a deregulated power system.

From the number of challenges and undeveloped areas that have been mentioned, this paper proposes a preventive maintenance scheduling model for WTs of an OWF in a deregulated power system through a bi-level optimization problem (BLP). In the lower-level problem, an economic dispatch is performed with the objective of minimizing the operation cost (maximizing social welfare) of the system. The constraints of the lower-level problem are the capacity of transmission lines, maximum available power through wind in the OWF and other power plants and the demand balance equation. In the upper-level problem, maintenance scheduling of all WTs in the offshore farm is carried out. Multiple constraints such as duration of each preventive maintenance action, the time required for each type of transfer vessel to reach the farm and return to the coast, the wave height 
limit for the vessels, the working shift limits, the wake effect and priority for some WTs are considered. The objective of the upper-level is to maximize the profit of the offshore farm, which is produced by multiplication of the electricity price into the produced power deducted by O\&M costs. The wake effect influencing the wind farm is added through the lower-level. Finally, the priority feature allows inclusion of condition monitoring signals into the maintenance scheduling. This BLP is reformulated in a single-level optimization problem (SLP) through Karush-Kuhn-Tucker (KKT) conditions.

\section{Problem Formulation}

Two maintenance scheduling models are proposed in this section. The first model, passive maintenance scheduling, aims to find the maintenance time windows, as well as their corresponding transport vessels by minimizing the operation cost of the power system where the OWF maintenance-related costs are also considered. In this model, the decisions are made by an independent system operator. The active maintenance scheduling model tries to solve this decision making problem through a profit-maximization perspective.

\subsection{Cost-Minimization Maintenance Scheduling}

For cost-minimization maintenance scheduling model, an SLP is defined as follows:

$$
\begin{aligned}
& \underset{q, u, y, z, \theta}{\operatorname{Minimize}} \sum_{h, g=O W F}\left[\sum_{s} \rho_{s}\left(f_{g} \alpha_{g} q_{h, g, s}+o_{g} q_{h, g, s}\right)+\sum_{k, b, r}\left(u_{h, k, b, r} m_{b}+z_{h, k, b, r}\right) C\right] \\
& +\sum_{h, g \neq O W F}\left[\sum_{s} \rho_{s}\left(f_{g} \alpha_{g} q_{h, g, s}+o_{g} q_{h, g, s}\right)+\sum_{r} y_{h, g, r} C\right] \\
& \sum_{h} y_{h, g, r}-T_{g}^{\prime \prime}=0 \\
& \forall g \neq O W F, r \\
& \sum_{g \neq O W F} y_{h, g, r}-1 \leq 0 \\
& \forall h, r \\
& y_{h+1, g, r}-y_{h, g, r}-y_{h+T_{g}^{\prime \prime}, g, r} \leq 0 \\
& \forall h, g \neq O W F, r \\
& \sum_{h, b} z_{h, k, b, r}-T_{k}=0 \\
& \forall k, r \\
& \sum_{k, b} z_{h, k, b, r}-1 \leq 0 \\
& \forall h, r \\
& \sum_{r} z_{h, k, b, r}-1 \leq 0 \\
& \forall h, k, b \\
& \sum_{k, b} u_{h, k, b, r}-1 \leq 0 \\
& \forall h, r \\
& \sum_{r} u_{h, k, b, r}-1 \leq 0 \\
& \forall h, k, b \\
& z_{h+1, k, b, r}-z_{h, k, b, r}-z_{h+T_{k}, k, b, r} \leq 0 \\
& \forall h, k, b, r \\
& z_{h, k, b, r}=0 \\
& z_{h, k, b, r}=0 \\
& z_{h, k, b, r} \leq u_{h, k, b, r} \\
& z_{h, k, b, r} \leq u_{h-T_{k, b}^{\prime}, k, b, r} \\
& z_{h, k, b, r} \leq u_{h+T_{k, b}^{\prime}, k, b, r} \\
& u_{h+1, k, b, r}-u_{h, k, b, r}-u_{h+T_{k}+2 T_{k, b}^{\prime}, k, b, r} \leq 0 \\
& \forall h<T_{k, b}^{\prime}, k, b, r \\
& \forall h>N_{h}-T_{k, b}^{\prime}, k, b, r \\
& \forall h, k, b, r \\
& \forall h>T_{k, b}^{\prime}, k, b, r \\
& \forall h<N_{h}-T_{k, b}^{\prime}, k, b, r \\
& \forall h, k, b, r \\
& \sum_{h, b} u_{h, k, b, r}-\left(2 T_{k, b}^{\prime}+T_{k}\right) \leq 0 \\
& \forall k, b=b_{1}, r \\
& \sum_{h, b} u_{h, k, b, r}-\left(2 T_{k, b}^{\prime}+T_{k}\right) \leq 0 \\
& \forall k, b=b_{2}, r
\end{aligned}
$$




$$
\begin{array}{ll}
\sum_{h, b} u_{h, k, b, r}-\left(2 T_{k, b}^{\prime}+T_{k}\right) \leq 0 & \forall k, b=b_{3}, r \\
\sum_{h, b} u_{h, k, b, r}-6 \leq 0 & \forall k, r \\
z_{h, k, b, r}=0 & \forall h \in \tau, k, b, r \\
z_{h, k, b, r}=0 & \forall h>h^{\prime}, k=k^{\prime}, b, r \\
w_{h, s}+\left(u_{h, k, b, r}-1\right) M_{1} \leq L_{b} & \forall h, k, b, s, r \\
-\sum_{g \in Y(i)} q_{h, g, s}+\sum_{j \in \Theta(i)} \frac{\left(\theta_{h, i, s}-\theta_{h, j, s}\right)}{X_{i, j}}+D_{h, i}=0 & \forall h, i, s \\
\frac{\left(\theta_{h, i, s}-\theta_{h, j, s}\right)}{X_{i, j}}-F_{i, j} \leq 0 & \\
q_{h, g, s}-\left(1-y_{h, g, r}\right) \bar{Q}_{g} \leq 0 & \forall h, i, j \mid j \in \Theta(i), s \\
q_{h, g, s}-\sum_{k}\left[\left(1-z_{h, k, b, r}\right) \bar{Q}_{h, g, k, s}^{\prime}\right] \leq 0 & \forall h, g \neq O W F, s, r \\
-q_{h, g, s} \leq 0 & \forall h, g=O W F, b, s, r
\end{array}
$$

The objective Function (1a) accounts for the operation cost of the system, as well as the maintenance action and vessel transportation costs. Constraint (1b) enforces all of the generators (except the OWF) to have their necessary required maintenance time. While Equation (1c) limits the maintenance action to only one generator at each hour, Equation (1d) defines the continuous maintenance operation. Constraint (1e) ensures that all WTs undergo maintenance for the required predefined maintenance time $T_{k}$. Constraints (1f) and (1h) make sure that for each hour, at most one maintenance action and one vessel are utilized, respectively. Constraints (1g) and (1i) help to distinguish among various maintenance actions. While Constraint (1j) guarantees a continuous maintenance action, Constraints (1k) and (11) provide enough time for the vessels to reach wind farm from the coast and return back, respectively.

A primary link between maintenance action and vessel operation is provided through Equation $(1 \mathrm{~m})$ where $u_{h, k, g, r}$ is triggered to become one whenever $z_{h, k, b, r}$ is one. Next, whenever $z_{h, k, b, r}$ is one, Constraints (1n) and (1o) correspondingly cause $u_{h, k, b, r}$ to be one, based on the vessel's transportation time. In other words, they consider the required time for the vessel to reach the wind farm and come back from the wind farm, respectively. Subsequently, Equation (1p) secures a continuous operation of the vessel transportation. Constraints (1q)-(1t) force the various vessel types to follow their predefined transportation time and do not exceed the overall specified time by limiting the operation of the vessels through each WT. It is assumed that the vessel remains at the WT's site during the maintenance time. It is also considered that the maintenance schedules are required to be performed only during specific time shifts through Equation (1u).

Constraint $(1 \mathrm{v})$ delivers the possibility of linking the model with a condition monitoring system and combining the information in the decision making process. For instance, if the information from the condition monitoring system expresses that a particular turbine $\left(k^{\prime}\right)$ will need maintenance shortly (in the next $h^{\prime}$ hours), these commands are implemented in the model through Constraint $(1 \mathrm{v})$. Even if a sudden change occurs in the middle of the time horizon, by fixing the already made decisions for other WTs, the model can run again solely for that particular WT. Moreover, each vessel has a wave height limit. Whenever the wave height is higher than the limit, the vessel cannot be used. Constraint $(1 \mathrm{w})$ applies this limitation. It should be mentioned that no limitation for the usage of the helicopter is considered.

Demand is supplied at each node in the system through Equation (1x), and the transmission capacity constraints are considered through Equation (1y). Each generator has a maximum capacity, and the maximum capacity of the wind farm is the sum of all available power that can be produced by 
each WT. The produced power of the generators should be less than these constraints and also positive, Equations (1z)-(1ab).

\subsection{Profit-Maximization Maintenance Scheduling}

For profit-maximization maintenance scheduling model, a BLP is derived as follows:

$$
\begin{aligned}
& \underset{u, y, z}{\operatorname{Maximize}} \sum_{h, g=O W F}\left[\sum_{s} \rho_{s}\left(\mu_{h, i, s} q_{h, g, s}-f_{g} \alpha_{g} q_{h, g, s}-o_{g} q_{h, g, s}\right)-\sum_{k, b, r}\left(u_{h, k, b, r} m_{b}+z_{h, k, b, r}\right) C\right] \\
& -\sum_{h, g \neq O W F}\left[\sum_{s} \rho_{s}\left(f_{g} \alpha_{g} q_{h, g, s}+o_{g} q_{h, g, s}\right)+\sum_{r} y_{h, g, r} C\right] \\
& \mu_{h, i, s}=\frac{1}{\rho_{s}} \lambda_{1_{h, i, s}} \\
& \sum_{h} y_{h, g, r}-T_{g}^{\prime \prime}=0 \\
& \sum_{g \neq O W F} y_{h, g, r}-1 \leq 0 \\
& y_{h+1, g, r}-y_{h, g, r}-y_{h+T_{g}^{\prime \prime}, g, r} \leq 0 \\
& \sum_{h, b} z_{h, k, b, r}-T_{k}=0 \\
& \sum_{k, b} z_{h, k, b, r}-1 \leq 0 \\
& \sum_{r} z_{h, k, b, r}-1 \leq 0 \\
& \sum_{k, b} u_{h, k, b, r}-1 \leq 0 \\
& \sum_{r} u_{h, k, b, r}-1 \leq 0 \\
& \forall h, i, s \\
& \forall g \neq O W F, r \\
& \forall h, r \\
& \forall h, g, r \\
& \forall k, r \\
& \forall h, r \\
& \forall h, k, b \\
& \forall h, r \\
& \forall h, k, b \\
& z_{h+1, k, b, r}-z_{h, k, b, r}-z_{h+T_{k}, k, b, r} \leq 0 \\
& \forall h, k, b, r \\
& z_{h, k, b, r}=0 \\
& \forall h<T_{k, b}^{\prime}, k, b, r \\
& z_{h, k, b, r}=0 \\
& z_{h, k, b, r} \leq u_{h, k, b, r} \\
& z_{h, k, b, r} \leq u_{h-T_{k, b}^{\prime}, k, b, r} \\
& z_{h, k, b, r} \leq u_{h+T_{k, b}^{\prime}, k, b, r} \\
& u_{h+1, k, b, r}-u_{h, k, b, r}-u_{h+T_{k}+2 T_{k, b}^{\prime}, k, b, r} \leq 0 \\
& \forall h>N_{h}-T_{k, b}^{\prime}, k, b, r \\
& \forall h, k, b, r \\
& \forall h>T_{k, b}^{\prime}, k, b, r \\
& \forall h<N_{h}-T_{k, b}^{\prime}, k, b, r \\
& \forall h, k, b, r \\
& \sum_{h, b} u_{h, k, b, r}-\left(2 T_{k, b}^{\prime}+T_{k}\right) \leq 0 \\
& \forall k, b=b_{1}, r \\
& \sum_{h, b} u_{h, k, b, r}-\left(2 T_{k, b}^{\prime}+T_{k}\right) \leq 0 \\
& \forall k, b=b_{2}, r \\
& \sum_{h, b} u_{h, k, b, r}-\left(2 T_{k, b}^{\prime}+T_{k}\right) \leq 0 \\
& \forall k, b=b_{3}, r \\
& \sum_{h, b} u_{h, k, b, r}-6 \leq 0 \\
& \forall k, r \\
& \forall h \in \tau, k, b, r \\
& \forall h>h^{\prime}, k=k^{\prime}, b, r \\
& z_{h, k, b, r}=0 \\
& \forall h, k, b, s, r \\
& \begin{array}{l}
w_{h, s}+\left(u_{h, k, b, r}-1\right) M_{1} \leq L_{b} \\
\underset{q, \theta}{\text { Minimize }} \sum_{h, g, s} \rho_{s}\left[f_{g} \alpha_{g} q_{h, g, s}+o_{g} q_{h, g, s}\right]
\end{array}
\end{aligned}
$$




$$
\begin{array}{ll}
-\sum_{g \in Y(i)} q_{h, g, s}+\sum_{j \in \Theta(i)} \frac{\left(\theta_{h, i, s}-\theta_{h, j, s}\right)}{X_{i, j}}+D_{h, i}=0: \lambda_{1_{h, i s}} & \forall h, i, s \\
\frac{\left(\theta_{h, i, s}-\theta_{h, j, s}\right)}{X_{i, j}}-F_{i, j} \leq 0: \lambda_{2_{h, i, j, s}} & \forall h, i, j \mid j \in \Theta(i), s \\
q_{h, g, s}-\left(1-y_{h, g, r}\right) \bar{Q}_{g} \leq 0: \lambda_{3_{h, g, s}} & \forall h, g \neq O W F, s, r \\
q_{h, g, s}-\sum_{k}\left[\left(1-z_{h, k, b, r}\right) \bar{Q}_{h, g, k, s}^{\prime}\right] \leq 0: \lambda_{4_{h, g, b s s}} & \forall h, g=O W F, b, s, r \\
-q_{h, g, s} \leq 0: \lambda_{5_{h, g, s}} & \forall h, g, s
\end{array}
$$

The upper-level problem, Equations (2a)-(2x), schedules the vessels and maintenance actions for each WT with the objective of maximizing the profit of the offshore farm. The lower-level problem, Equations (2y)-(2ad), performs an economic dispatch in the system with the objective of minimizing operation costs.

The objective Function (2a) is the profit of the wind farm minus the operation and maintenance action costs of other generating units. The terms related to other generating units are included here as they significantly reduce the required computing power and facilitate reaching an optimum solution. Please notice that the drawback of this inclusion of variables is that it transforms the problem into a cooperative maintenance scheduling. On the other hand, in order to evaluate this cooperative matter, it has been addressed in one of the case studies. Indeed, if more computing power is available, one can further study the proposed strategic maintenance scheduling of offshore wind farms (SMSOWF) model by solely focusing on the non-cooperative perspective. The first term, $\mu_{h, i, s} q_{h, g, s}$, is the revenue that comes from multiplication of the electricity price (locational marginal price, $\mu_{h, i, s}$ ) into the production of the wind farm, $q_{h, g, s}$. Please note that $\mu_{h, i, s}$ is obtained through using the dual variable of the demand balance, $\lambda_{1_{h, i, s}}$ in Equation (2z). The second and third terms, $f_{g} \alpha_{g} q_{h, g, s}$ and $o_{g} q_{h, g, s}$, account for operation costs. The fourth and fifth terms, $\left(u_{h, k, b, r} m_{b}+z_{h, k, b, r}\right) C$, build the costs regarding the maintenance actions and vessels' transportation. The last terms, $q_{h, g, s}+o_{g} q_{h, g, s}+y_{h, g, r} C$, consider the operation and maintenance-related costs of generating units, except the wind farm. The objective function of the lower-level problem, Equation (2y), consists of costs related to the operation cost of the system where a minimization would result in the least operation cost. $\lambda$ values are the Lagrange multipliers of their corresponding equations in the lower-level problem. It should be noted that in the profit-maximization model where the profit of the OWF is maximized, the scheduling of all of the power plants is included. Such consideration offers the evaluation of strategic operations. Moreover, a comparison between Case III (where the maintenance schedule of all power plants, except the OWF, is decided by the independent system operator) and other cases (where such consideration is relaxed) delivers insight into different perspectives in the operation and maintenance planning.

\subsection{Karush-Kuhn-Tucker Conditions}

The profit-maximization model formulated in Equation (2) is a bi-level problem. It should be noted that since the unit commitment decisions (start-up and shut-down) are not directly considered, the lower-level problem, Equations (2y)-(2ad), is convex. Therefore, the BLP can be transformed into an SLP through KKT conditions. KKT conditions include stationarity (gradient of Lagrangian function $(\mathcal{L})$ with respect to lower-level variables), complementary slackness, primal (original inequalities) and dual constraints [32]. These should be derived from the lower-level problem. The following shows the Lagrangian function:

$$
\begin{aligned}
& \mathcal{L}(q, \theta, \lambda)=\sum_{h, g, s} \rho_{s}\left[f_{g} \alpha_{g} q_{h, g, s}+o_{g} q_{h, g, s}\right]+\sum_{h, i, s} \lambda_{1_{h, i s}}\left[\sum_{g \in Y(i)}-q_{h, g, s}+\sum_{j \in \Theta(i)} \frac{\left(\theta_{h, i, s}-\theta_{h, j, s}\right)}{X_{i, j}}+D_{h, i}\right] \\
& +\sum_{h, i, j \in \Theta(i), s} \lambda_{2_{h, i, j, s}}\left[\frac{\left(\theta_{h, i, s}-\theta_{h, j, s}\right)}{X_{i, j}}-F_{i, j}\right]+\sum_{h, g \neq O W F, s, r} \lambda_{3_{h, g, s}}\left[q_{h, g, s}-\left(1-y_{h, g, r}\right) \bar{Q}_{g}\right]
\end{aligned}
$$




$$
+\sum_{h, g=O W F, b, s, r} \lambda_{4_{h, g, b, s}}\left[q_{h, g, s}-\sum_{k}\left[\left(1-z_{h, k, b, r}\right) \bar{Q}_{h, g, k, s}^{\prime}\right]\right]+\sum_{h, g, s} \lambda_{5_{h, g, s}}\left(-q_{h, g, s}\right)
$$

From stationarity conditions:

$$
\begin{array}{ll}
\rho_{s}\left(f_{g} \alpha_{g}+o_{g}\right)-\lambda_{1_{h, i, s}}+\lambda_{3_{h, g, s}}-\lambda_{5_{h, g, s}}=0 & \forall h, g \neq O W F, s \\
\rho_{s}\left(f_{g} \alpha_{g}+o_{g}\right)-\lambda_{1_{h, i, s}}+\sum_{b} \lambda_{4_{h, g, b s}}-\lambda_{5_{h, g, s}}=0 & \forall h, g=O W F, s \\
\sum_{j \in \Theta(i)} \frac{\left(\lambda_{1_{h, i, s}}-\lambda_{1_{h, j, s}}\right)}{X_{i, j}}+\sum_{j \in \Theta(i)} \frac{\left(\lambda_{2_{h, i, j, s}}-\lambda_{2_{h, j, i, s}}\right)}{X_{i, j}}=0 & \forall h, i, s
\end{array}
$$

Additionally, by writing complementarity conditions:

$$
\begin{array}{ll}
\lambda_{2_{h, i, j, s}}\left[\frac{\left(\theta_{h, i, s}-\theta_{h, j, s}\right)}{X_{i, j}}-F_{i, j}\right]=0 & \forall h, i, j \in \Theta(i), s \\
\lambda_{3_{h, g, s}}\left[q_{h, g, s}-\left(1-y_{h, g, r}\right) \bar{Q}_{g}\right]=0 & \forall h, g \neq O W F, s, r \\
\lambda_{4_{h, g, b, s}}\left[q_{h, g, s}-\sum_{k}\left[\left(1-z_{h, k, b, r}\right) \bar{Q}_{h, g, k, s}^{\prime}\right]\right]=0 & \forall h, g=O W F, b, s, r \\
\lambda_{5_{h, g, s}}\left(-q_{h, g, s}\right)=0 & \forall h, g, s
\end{array}
$$

The complementarity conditions, Equations (7)-(10), and the objective Function (2a) include nonlinearities. In this regard, the complementarity conditions can be substituted by the strong duality as follows:

$$
\begin{aligned}
& \sum_{h, g, s} \rho_{s}\left[f_{g} \alpha_{g} q_{h, g, s}+o_{g} q_{h, g, s}\right]=\sum_{h, i, s} \lambda_{1_{h, i, s}} D_{h, i}-\sum_{h, i, j \in \Theta(i), s} \lambda_{2_{h, i, j, s}} F_{i, j}-\sum_{h, g \neq O W F, s, r} \lambda_{3_{h, g, s}}\left(1-y_{h, g, r}\right) \bar{Q}_{g} \\
& -\sum_{h, g=O W F, b, s, r} \lambda_{4_{h, g, b, s}}\left[\sum_{k}\left(1-z_{h, k, b, r}\right) \bar{Q}_{h, g, k, s}^{\prime}\right]
\end{aligned}
$$

There are two nonlinear terms in the strong duality. The nonlinear term $\lambda_{4_{h, g, b s}}\left(1-z_{h, k, b, r}\right)$ can be linearized as:

$$
\begin{array}{ll}
\lambda_{h, g, k, b, s}^{\prime}=\lambda_{4_{h, g, b, s}}\left(1-\sum_{r} z_{h, k, b, r}\right) & \forall h, g=O W F, k, b, s \\
\lambda_{h, g, k, b, s}^{\prime} \leq\left(1-\sum_{r} z_{h, k, b, r}\right) M_{2} & \forall h, g=O W F, k, b, s \\
\lambda_{4_{h, g, b, s}}-\lambda_{h, g, k, b, s}^{\prime} \leq\left[1-\left(1-\sum_{r} z_{h, k, b, r}\right)\right] M_{2} & \forall h, g=O W F, k, b, s \\
\lambda_{4_{h, g, b, s}}-\lambda_{h, g, k, b, s}^{\prime} \geq-\left[1-\left(1-\sum_{r} z_{h, k, b, r}\right)\right] M_{2} & \forall h, g=O W F, k, b, s
\end{array}
$$

and the nonlinear term $\lambda_{3_{h, g, s}}\left(1-y_{h, g, r}\right)$ can be linearized as:

$$
\begin{array}{ll}
\lambda_{h, g, s}^{\prime \prime}=\lambda_{3_{h, g, s}}\left(1-\sum_{r} y_{h, g, r}\right) & \forall h, g \neq O W F, s \\
\lambda_{h, g, s}^{\prime \prime} \leq\left(1-\sum_{r} y_{h, g, r}\right) M_{3} & \forall h, g \neq O W F, s \\
\lambda_{3_{h, g, s}}-\lambda_{h, g, s}^{\prime \prime} \leq\left[1-\left(1-\sum_{r} y_{h, g, r}\right)\right] M_{3} & \forall h, g \neq O W F, s \\
\lambda_{3 h, g, s}-\lambda_{h, g, s}^{\prime \prime} \geq-\left[1-\left(1-\sum_{r} y_{h, g, r}\right)\right] M_{3} & \forall h, g \neq O W F, s
\end{array}
$$


where $M_{2}$ and $M_{3}$ are large enough positive constants [33]. Thus, the strong duality renders into:

$$
\begin{aligned}
& \sum_{h, g, s} \rho_{s}\left[f_{g} \alpha_{g} q_{h, g, s}+o_{g} q_{h, g, s}\right]=\sum_{h, i, s} \lambda_{1_{h, i, s}} D_{h, i}-\sum_{h, i, j \in \Theta(i), s} \lambda_{2_{h, i, j, s}} F_{i, j}-\sum_{h, g \neq O W F, s} \lambda_{h, g, s}^{\prime \prime} \bar{Q}_{g} \\
& -\sum_{h, g=O W F, k, b, s} \lambda_{h, g, k, b, s}^{\prime} \bar{Q}_{h, g, k, s}^{\prime}
\end{aligned}
$$

To linearize the term $\lambda_{1_{h, i,}} q_{h, g, s}$ in the objective Function (2a), stationarity Condition (5) is multiplied by $q_{h, g, s}$ as follows:

$$
\rho_{s}\left(f_{g} \alpha_{g} q_{h, g, s}+o_{g} q_{h, g, s}\right)-\lambda_{1_{h, i, s}} q_{h, g, s}+\sum_{b} \lambda_{4_{h, g, b, s}} q_{h, g, s}-\lambda_{5_{h, g, s}} q_{h, g, s}=0 \quad \forall h, g=O W F, s
$$

The last term in Equation (21) should then be omitted due to Equation (10). The nonlinear term $\lambda_{4_{h, g, b s}} q_{h, g, s}$ in Equation (21) can also be simplified through Equation (9). In addition, by considering Equation (12), Equation (21) becomes:

$$
\lambda_{\substack{1_{h, i, s} \\ i \leftarrow g}} q_{h, g, s}=\rho_{s}\left(f_{g} \alpha_{g} q_{h, g, s}+o_{g} q_{h, g, s}\right)+\sum_{k, b, s} \lambda_{h, g, k, b, s}^{\prime} \bar{Q}_{h, g, k, s}^{\prime} \quad \forall h, g=O W F, s
$$

which is the linear equivalent of the nonlinear term in the objective Function (2a).

Those steps convert the BLP in Equation (2) into an SLP as follows:

$$
\begin{array}{ll}
\operatorname{Maximize}_{q, u, y, z, \theta} \sum_{h, g=O W F, k, b}\left[\sum_{s} \rho_{s} \lambda_{h, g, k, b, s}^{\prime} \bar{Q}_{h, g, k, s}^{\prime}-\sum_{r}\left(u_{h, k, b, r} m_{b}+z_{h, k, b, r}\right) C\right] \\
-\sum_{h, g \neq O W F}\left[\sum_{s} \rho_{s}\left(f_{g} \alpha_{g} q_{h, g, s}+o_{g} q_{h, g, s}\right)+\sum_{r} y_{h, g, r} C\right] \\
\mu_{h, i, s}=\frac{1}{\rho_{s}} \lambda_{1_{h, i, s}} & \forall h, i, s \\
\sum_{h} y_{h, g, r}-T_{g}^{\prime \prime}=0 & \forall g \neq O W F, r \\
\sum_{g \neq O W F} y_{h, g, r}-1 \leq 0 & \forall h, r \\
y_{h+1, g, r}-y_{h, g, r}-y_{h+T_{g}^{\prime \prime}, g, r} \leq 0 & \forall h, g, r \\
\sum_{h, b} z_{h, k, b, r}-T_{k}=0 & \forall k, r \\
\sum_{k, b} z_{h, k, b, r}-1 \leq 0 & \forall h, r \\
\sum_{r} z_{h, k, b, r}-1 \leq 0 & \forall h, k, b \\
\sum_{k, b} u_{h, k, b, r}-1 \leq 0 & \forall h, r \\
\sum_{r} u_{h, k, b, r}-1 \leq 0 & \forall h, k, b \\
z_{h+1, k, b, r}-z_{h, k, b, r}-z_{h+T_{k}, k, b, r} \leq 0 & \forall h, k, b, r \\
z_{h, k, b, r}=0 & \forall h<T_{k, b}^{\prime}, k, b, r \\
z_{h, k, b, r}=0 & \forall h>N_{h}-T_{k, b}^{\prime}, k, b, r \\
z_{h, k, b, r} \leq u_{h, k, b, r} & \forall h, k, b, r \\
z_{h, k, b, r} \leq u_{h-T_{k, b}^{\prime}, k, b, r} & \forall h>T_{k, b}^{\prime}, k, b, r \\
z_{h, k, b, r} \leq u_{h+T_{k, b}^{\prime}, k, b, r} & \forall h<N_{h}-T_{k, b}^{\prime}, k, b, r \\
&
\end{array}
$$




$$
\begin{aligned}
& u_{h+1, k, b, r}-u_{h, k, b, r}-u_{h+T_{k}+2 T_{k, b}^{\prime}, k, b, r} \leq 0 \\
& \forall h, k, b, r \\
& \sum_{h, b} u_{h, k, b, r}-\left(2 T_{k, b}^{\prime}+T_{k}\right) \leq 0 \\
& \forall k, b=b_{1}, r \\
& \sum_{h, b} u_{h, k, b, r}-\left(2 T_{k, b}^{\prime}+T_{k}\right) \leq 0 \\
& \forall k, b=b_{2}, r \\
& \sum_{h, b} u_{h, k, b, r}-\left(2 T_{k, b}^{\prime}+T_{k}\right) \leq 0 \\
& \forall k, b=b_{3}, r \\
& \sum_{h, b} u_{h, k, b, r}-6 \leq 0 \\
& \forall k, r \\
& \forall h \in \tau, k, b, r \\
& z_{h, k, b, r}=0 \\
& \forall h>h^{\prime}, k=k^{\prime}, b, r \\
& \forall h, k, b, s, r \\
& w_{h, s}+\left(u_{h, k, b, r}-1\right) M_{1} \leq L_{b} \\
& -\sum_{g \in \mathrm{Y}(i)} q_{h, g, s}+\sum_{j \in \Theta(i)} \frac{\left(\theta_{h, i, s}-\theta_{h, j, s}\right)}{X_{i, j}}+D_{h, i}=0 \\
& \forall h, i, s \\
& \frac{\left(\theta_{h, i, s}-\theta_{h, j, s}\right)}{X_{i, j}}-F_{i, j} \leq 0 \\
& q_{h, g, s}-\left(1-y_{h, g, r}\right) \bar{Q}_{g} \leq 0 \\
& q_{h, g, s}-\sum_{k}\left[\left(1-z_{h, k, b, r}\right) \bar{Q}_{h, g, k, s}^{\prime}\right] \leq 0 \\
& -q_{h, g, s} \leq 0 \\
& \rho_{s}\left(f_{g} \alpha_{g}+o_{g}\right)-\underset{\lambda_{h, i, s}}{i \leftarrow g}-\lambda_{3_{h, g, s}}-\lambda_{5_{h, g, s}}=0 \\
& \rho_{s}\left(f_{g} \alpha_{g}+o_{g}\right)-\underset{\substack{\lambda_{h i, s} \\
i \leftarrow g}}{\lambda_{b}}+\sum_{b} \lambda_{4_{h, g, b, s}}-\lambda_{5_{h, g, s}}=0 \\
& \sum_{j \in \Theta(i)} \frac{\left(\lambda_{1_{h, i s}}-\lambda_{1_{h, j, s}}\right)}{X_{i, j}}+\sum_{j \in \Theta(i)} \frac{\left(\lambda_{2_{h, i, j, s}}-\lambda_{2_{h, j, i, s}}\right)}{X_{i, j}}=0 \\
& \sum_{h, g, s} \rho_{s}\left[f_{g} \alpha_{g} q_{h, g, s}+o_{g} q_{h, g, s}\right]=\sum_{h, i, s} \lambda_{1_{h, i, s}} D_{h, i}-\sum_{h, i, j \in \Theta(i), s} \lambda_{2_{h, i, j, s}} F_{i, j} \\
& -\sum_{h, g \neq O W F, s} \lambda_{h, g, s}^{\prime \prime} \bar{Q}_{g}-\sum_{h, g=O W F, k, b, s} \lambda_{h, g, k, b, s}^{\prime} \bar{Q}_{h, g, k, s}^{\prime} \\
& \lambda_{h, g, k, b, s}^{\prime} \leq\left(1-\sum_{r} z_{h, k, b, r}\right) M_{2} \\
& \lambda_{4_{h, g, b, s}}-\lambda_{h, g, k, b, s}^{\prime} \leq\left[1-\left(1-\sum_{r} z_{h, k, b, r}\right)\right] M_{2} \\
& \lambda_{4_{h, g, b, s}}-\lambda_{h, g, k, b, s}^{\prime} \geq-\left[1-\left(1-\sum_{r} z_{h, k, b, r}\right)\right] M_{2} \\
& \lambda_{h, g, s}^{\prime \prime} \leq\left(1-\sum_{r} y_{h, g, r}\right) M_{3} \\
& \lambda_{3_{h, g, s}}-\lambda_{h, g, s}^{\prime \prime} \leq\left[1-\left(1-\sum_{r} y_{h, g, r}\right)\right] M_{3} \\
& \lambda_{3_{h, g, s}}-\lambda_{h, g, s}^{\prime \prime} \geq-\left[1-\left(1-\sum_{r} y_{h, g, r}\right)\right] M_{3}
\end{aligned}
$$

\section{Case Study}

\subsection{Test System}

The proposed SMSOWF model in Equation (23) is coded in GAMS v24.7.1 [34] and solved using CPLEX v12.6.2 [35] on a machine running with 144 GB of RAM and an Intel Xeon E5-2660 (2.6 GHz) 
with two processors (20 cores). A five-bus network (Figure 1 ) is defined to demonstrate the applicability of the developed SMSOWF model. Details of the generators are provided in Table 1. The inductance and capacity of all transmission lines are correspondingly $0.06 \Omega$ and $60 \mathrm{MW}$. The demand profit is shown in Figure 2. The considered operating horizon is for $200 \mathrm{~h}$, where it can be scaled up or modified as required based on days, weeks, etc.

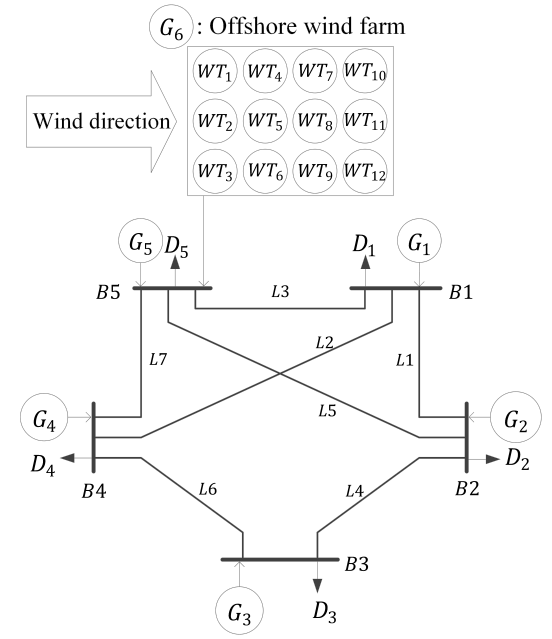

Figure 1. The utilized five-bus test system.

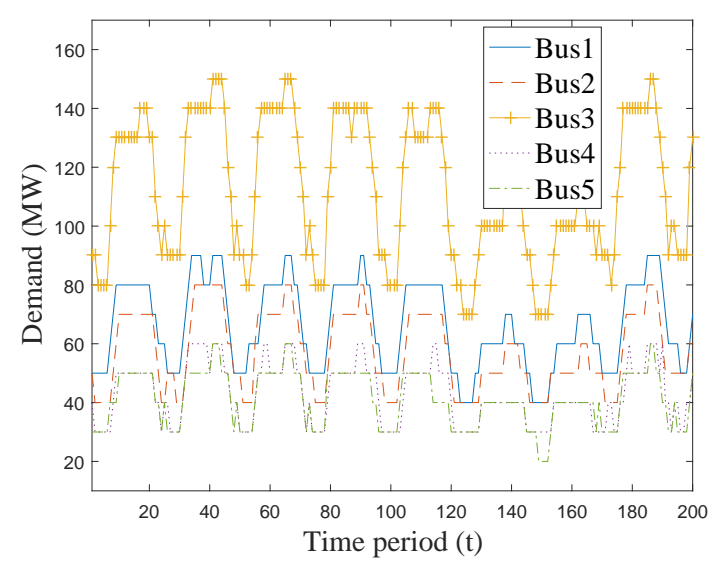

Figure 2. Load profile in the test system.

Two boats and a helicopter are considered as transportation mediums (vessels) for performing the maintenance actions. The vessels are differentiated by transportation time (Table 2), operation cost and wave height limitations (Table 3). The helicopter can travel the distance faster than the boats, has the highest cost and no wave height limit. The maintenance actions are allowed to be carried out only between 05:00 a.m. and 08:00 p.m. as the day working shift.

This paper also considers a simplified version of the wake effect in the maintenance scheduling problem. Wind direction is assumed fixed and shown in Figure 1 (wind blows from the west to the east). To add the wake effect, it is considered that the available wind power of each column of WTs reduces as the wind travels through the farm based on the wind direction. Thus, it is assumed that $W T_{1}, W T_{2}, W T_{3}$ have the hourly maximum available wind power based on Figure 3 . This maximum reduces by one unit for $W T_{4}, W T_{5}, W T_{6}$, by two units for $W T_{7}, W T_{8}, W T_{9}$ and by three units for $W T_{10}, W T_{11}, W T_{12}$, as displayed in Table 2. These are implemented in the model through the $\bar{Q}_{h, g, k}^{\prime}$ parameter. The wind and wave data can be found online [36], where for simplicity, the wind speed is approximated as output power, as illustrated in Figure 3. To account for variations in the wind and wave in the environment, 
stochasticity is also considered for them. In this regard, the variations range from $-3-+3$ standard deviations through seven levels (L1-L7) for each of the wind and wave data. The probability of each level is illustrated as in Figure 4. It should be mentioned that the reference data in the stochastic case (L4) for wind and wave are the data in Figure 3 where the considered standard deviations are 1.00 and 0.15 units for each of the wind and wave data, respectively.

Table 1. Operational and maintenance information of the generators.

\begin{tabular}{ccccc}
\hline Generator & $\bar{Q}_{g}\left({\overline{Q_{h, g}, k, s}}^{\prime}{ }^{a}\right)$ & $\alpha_{g}$ & $f_{g}$ & $\boldsymbol{o}_{g}$ \\
\hline G1 & 100 & 0.3 & 5 & 7 \\
G2 & 90 & 0.5 & 10 & 9 \\
G3 & 160 & 0.7 & 15 & 11 \\
G4 & 80 & 0.9 & 20 & 13 \\
G5 & 70 & 1.1 & 25 & 15 \\
G6 & $108^{\text {a }}$ & 0 & 0 & 10 \\
\hline
\end{tabular}

a Set based on Figure 3 and the reference in stochastic scenarios.

Table 2. Vessel transfer time, wake effect and forced outage rate (FOR).

\begin{tabular}{cccccc}
\hline \multirow{2}{*}{ Wind Turbine } & \multicolumn{2}{c}{ Vessel Transfer Time (h) } & Wake Effect (MW) & FOR \\
& $\boldsymbol{b}_{\mathbf{1}}$ & $\boldsymbol{b}_{\mathbf{2}}$ & $\boldsymbol{b}_{\mathbf{3}}$ & (Hourly Measure) & (p.u.) \\
\hline$W T_{1}, W T_{2}, W T_{3}$ & 2 & 1 & 0 & Figure 3 & $\mathbf{0 . 0 2}$ \\
$W T_{4}, W T_{5}, W T_{6}$ & 2 & 1 & 0 & Figure 3; 1 & $\mathbf{0 . 0 2}$ \\
$W T_{7}, W T_{8}, W T_{9}$ & 2 & 1 & 0 & Figure 3; 2 & $\mathbf{0 . 0 1}$ \\
$W T_{10}, W T_{11}, W T_{12}$ & 2 & 1 & 0 & Figure 3; 3 & $\mathbf{0 . 0 1}$ \\
\hline
\end{tabular}

Table 3. Vessel cost and transportation limit.

\begin{tabular}{ccc}
\hline \multirow{2}{*}{ Vessel } & $\begin{array}{c}\text { Vessel Cost Factor } \\
\boldsymbol{m}_{\boldsymbol{b}}\end{array}$ & $\begin{array}{c}\text { Wave Height Limit (m) } \\
\boldsymbol{L}_{\boldsymbol{b}}\end{array}$ \\
\hline$b_{1}$ & 0.1 & 0.5 \\
$b_{2}$ & 0.2 & 1.5 \\
$b_{3}$ & 0.8 & - \\
\hline
\end{tabular}

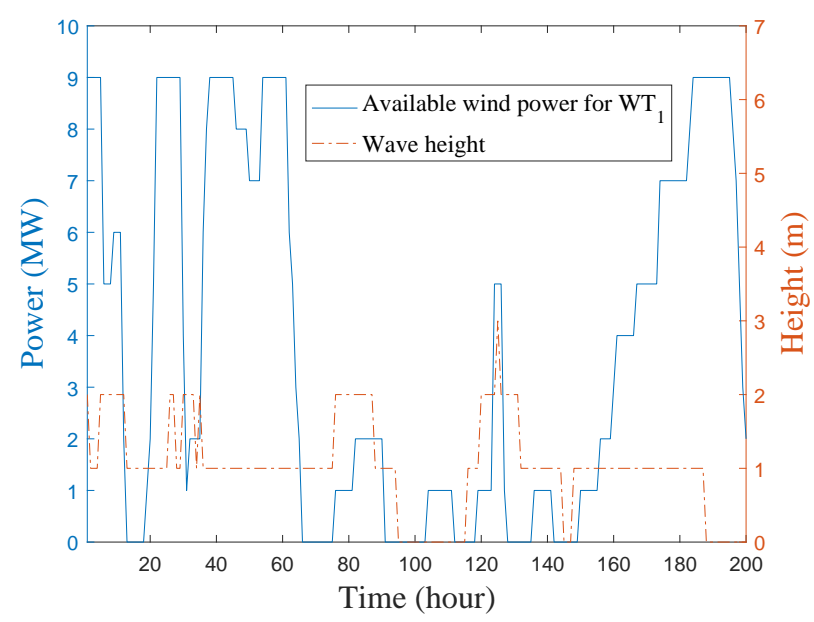

Figure 3. Available wind power and wave height in deterministic cases.

After developments and advancements in condition monitoring systems of WTs, the wind farm operators can receive signals regarding the real-time health condition of the WTs. Assuming the signal 
indicates an approaching failure, the operator should schedule a preventive maintenance action as soon as possible. This issue puts that particular WT in front of the line for receiving the maintenance and assigns a priority to that WT. For instance, through the SCADA system, it is assumed that $W T_{1}$ has received an alarm where it expresses that if $W T_{1}$ does not have a maintenance in the following two days ( $48 \mathrm{~h})$, it will fail.

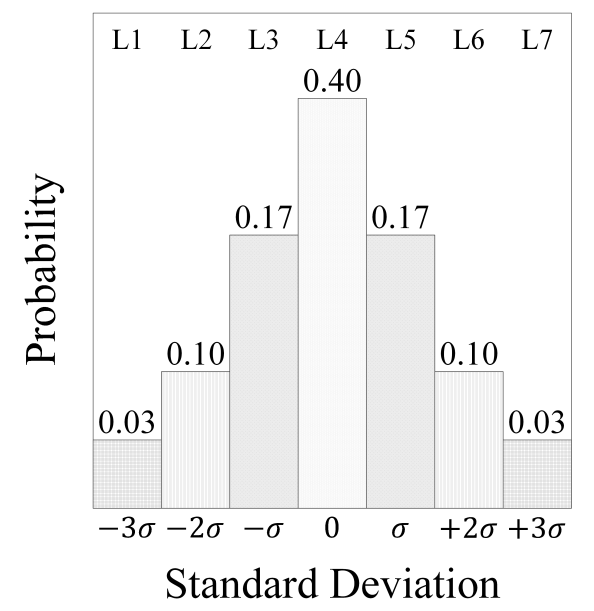

Figure 4. Stochasticity defined for wind and wave.

\subsection{Cases}

In order to demonstrate features and applicability of the developed SMSOWF model, nine cases are defined.

\subsubsection{Case I}

This case introduces a cost-minimization problem where the maintenance scheduling is performed for all of the power plants with the objective of achieving the least operation cost for the test system through Equation (1).

\subsubsection{Case II}

This case introduces a profit-maximization problem where the best time window for the preventive maintenance schedule of each WT in the offshore farm along with its corresponding transport vessel is found through Equation (23). In addition, the maintenance schedule for other generators is also decided. The objective is that these decisions should be in line with each other, so that the profit of the offshore farm is maximized.

\subsubsection{Case III}

In this case, the maintenance schedules of all power plants (except the OWF) that are obtained in Case I are considered the same ( $y_{h, g}$ is fixed for $G_{1}$ to $\left.G_{5}\right)$. Then, a profit-maximization problem is carried out for the OWF through Equation (23).

\subsubsection{Case IV}

Case IV proposes a case where after receiving the failure (or anomaly) alarm, preventive maintenance of all WTs is re-scheduled (assuming WTs did not have their preventive maintenance performed by that time), and the maintenance of the $W T_{1}$ is prioritized. However, the maintenance of other power plants is already decided ( $y_{h, g}$ is fixed for $G_{1}$ to $G_{5}$ from Case II).

In order to demonstrate the possibility of the integration of condition monitoring systems with the maintenance planning, a simple anomaly model is defined as in Figure 5. The anomaly model receives 
the health condition of the WT (e.g., from a SCADA system) and updates the health condition indicator that displays the status of the WT. In this example, 0.00 means the WT is in a good operational state, and 1.00 means the WT is in an anomaly or failure state. Due to the high costs of anomaly and failure conditions, the OWF operator would like to prevent any WT from reaching the failure state. Therefore, whenever the health condition indicator reaches a predefined threshold, $\Delta$ (which can be set based on experience), it is assumed that the WT must undergo maintenance by the time $t_{F}$. Otherwise, the WT may face an anomaly or a failure. Interested readers can refer to $[12,14,37]$ for detailed analyses on the developments of such condition monitoring systems and anomaly models, as a further look into this area is out of the scope of this paper.

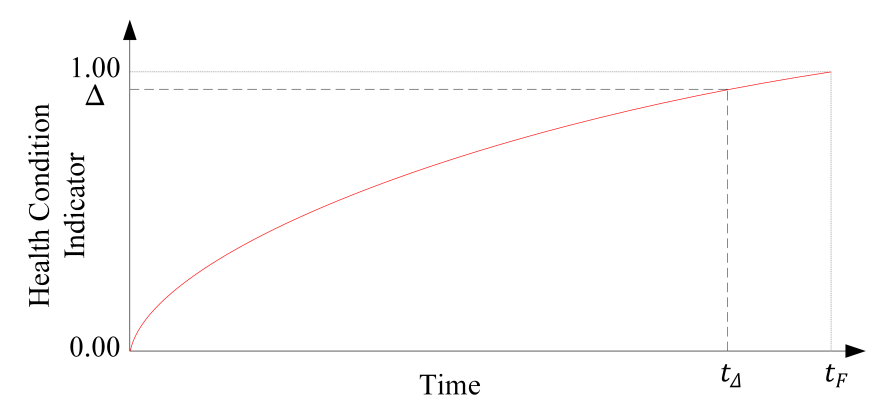

Figure 5. The defined anomaly model from the condition monitoring system.

\subsubsection{Case $\mathrm{V}$}

Case $\mathrm{V}$ assumes that $W T_{1}$ requires a prioritized action, and planning of preventive maintenance schedules for other WTs is already outlined ( $y_{h, g}$ is fixed for $G_{1}$ to $G_{5} ; z_{h, k, b}$ and $u_{h, k, b}$ are fixed for $W T_{2}$ to $W T_{12}$ from Case II). Hence, only the preventive maintenance of $W T_{1}$ needs to be re-scheduled to an earlier time within the next two days after receiving the alarm. It should be noted that the priority constraint, Equation ( $23 \mathrm{w})$, is only considered in Case IV and Case V $\left(h^{\prime}=48, k^{\prime}=W T_{1}\right)$. Based on Figure 5 , in this example, $t_{F}-t_{\Delta}$ can be considered as the remaining time for the WT until it faces an anomaly or failure in $h^{\prime}=48 \mathrm{~h}$, since the time it received the anomaly signal.

\subsubsection{Case VI}

In order to show the difference that addition of wake effect delivers, Case VI neglects the impact of the wake effect where it is assumed that available producible power for all of the WTs follows the same pattern depicted in Figure 3. Then, a profit-maximization problem is carried out through Equation (23).

\subsubsection{Case VII}

This case modifies Case II and considers the model without night work-shift limitation, which provides the possibility of performing maintenance at any hour of the day. To this aim, Equation (23v) is disregarded in this case, as it only enforces the day shift.

\subsubsection{Case VIII}

To account for the unexpected failure in the study, Case III is updated where the forced outage rate (FOR) is considered, as well. The assumed FOR values are displayed in Table 2 [38,39]. Moreover, since the wake effect can impact the performance of the WTs as the loading on them increases, the FOR for the WTs that are affected more by the wake effect is higher than the other WTs. The calculation of unexpected outage times based on FOR for the WTs is performed through the Monte Carlo technique where 2000 scenarios were simulated and one scenario was randomly selected to show the unexpected outage period for each WT based on its considered FOR. For simplicity, the FOR is solely considered for the WTs, and only the deterministic study is carried out for this case. 


\subsubsection{Case IX}

There may be a situation where the WTs or generators require more than one maintenance action (e.g., on different components). Such a possibility is also implemented in the model by updating Case $\mathrm{VI}$, and its application is demonstrated through Case IX. For simplicity, only the deterministic study is carried out, and the number of required maintenance actions is set to two, $N_{r}=2$, for each generator and WT.

\section{Results}

The profit of the offshore farm in each case is exhibited in Table 4. In the deterministic study, a comparison between Case I and Case II shows that there is a large potential for the offshore farm to increase its profit by participating in the market and considering the electricity prices in the decision making process. In other words, if the wind farm decides to plan its WTs preventive maintenance by considering the actual electricity prices, it could increase its profit by more than double. On the other hand, Case III shows that if the offshore farm cannot affect other power plants' maintenance schedules, it could still increase its profit by about $11 \%$.

Interestingly, it can be seen that in the stochastic study, the profits follow the same trend as in the deterministic study. It should also be mentioned that the maximum available power in the stochastic study (144) is more than in the deterministic study (108). This is the main reason of rather high profits in comparison with the deterministic cases. Another important point that must be mentioned here is that this addition of power in the stochastic case can present the OWF with more capability in influencing the market (prices, costs, etc.). Hence, the analysis of the strategic behavior is more prominent in the stochastic study over the deterministic one.

Case IV and Case V are of particular importance, since they directly integrate the performance analysis and anomaly detection information extracted from the SCADA system of the WT into the operation and decision making process. As expected, the profits in Table 4 show that if a sudden anomaly occurs and a preventive maintenance action is required, the best option (from the profit-maximization perspective) would be to re-schedule the maintenance of all of the WTs in the farm, Case IV. This is due to the reason that in the Case II, the $W T_{1}$ was scheduled for maintenance through vessel type $b_{1}$ (which is the lease expensive vessel). In Case IV, all schedules were re-arranged, and other WTs were able to use the possible periods to use the inexpensive vessel $b_{1}$. In Case V, since there was no available period for using vessel $b_{1}$ (in the $48 \mathrm{~h}$ after the alarm), vessel $b_{2}$ was ordered to be used in the emergency maintenance situation, which caused higher costs and, thus, lower profit for the OWF. On the other hand, it can be seen that the difference in the profit reduces in the stochastic study, and the impact is less. This means that in a more realistic case, due to the uncertainty in wind and wave, in such conditions, there is less opportunity for profiting in comparison with the deterministic study.

Table 4. Summary of profit and vessel utilization; deterministic (stochastic).

\begin{tabular}{|c|c|c|c|c|}
\hline & \multicolumn{3}{|c|}{ Number of Vessel Utilization } & \multirow{2}{*}{ Profit (\$) } \\
\hline & $b 1$ & $b 2$ & $b 3$ & \\
\hline Case I & $3(2)$ & $9(10)$ & $0(0)$ & 4716 (9875) \\
\hline Case II & $3(3)$ & $9(9)$ & $0(0)$ & $10,769(\mathbf{1 1}, \mathbf{4 1 0})$ \\
\hline Case III & $3(2)$ & $9(7)$ & $0(3)$ & $5220(\mathbf{1 0 , 4 2 3 )}$ \\
\hline Case IV & $3(2)$ & $9(10)$ & $0(0)$ & $10,483(\mathbf{1 0 , 6 3 2 )}$ \\
\hline Case V & $2(2)$ & $10(\mathbf{1 0})$ & $0(0)$ & $9810(\mathbf{1 0 , 6 0 7 )}$ \\
\hline Case VI & $3(2)$ & $9(\mathbf{1 0})$ & $0(0)$ & $28,204(25,489)$ \\
\hline Case VII & $4(4)$ & $8(8)$ & $0(0)$ & $10,867(\mathbf{1 7}, \mathbf{1 0 4})$ \\
\hline Case VIII & $3(-)$ & $9(-)$ & $0(-)$ & $3498(-)$ \\
\hline Case IX & $6(-)$ & $18(-)$ & $0(-)$ & $14,954(-)$ \\
\hline
\end{tabular}


On consideration of the wake effect in the analyses, while Case VI neglects the influence of wake effect, Case VII disregards the impact of daily working shift. Interestingly, the results show that ignoring the wake effect brings about outcomes that are very different from when the wake effect is considered (Table 4). Comparing Case II with Case VI shows that by neglecting the wake effect, the earned profit is $\$ 28,204$, which is nearly three-times (two-times in the stochastic study) more than the profit when the wake effect is considered. This is very important, as it can result in a planning strategy where it is far from the reality.

Regarding the work shifts, the results show that providing the possibility of carrying out maintenance at any hour of the day does not increase the profit significantly (Table 4). For instance, by providing the 24/7 maintenance crew in Case VII, the profit has only increased around $1 \%$ when compared with Case II with only daily working shift. It should be reminded that this change is more bold in the stochastic study as the maximum available wind power has increased. On the other hand, having an around-the-clock maintenance crew results in higher costs, which then will reduce the additionally earned profit. Furthermore, the proposed SMSOWF model provides a way to study and find the optimum daily work shift for the maintenance crew, which can be of great assistance for the asset management and maintenance department.

Table 5 displays the average electricity price and the operation cost of the system. It should be mentioned that the operation cost is defined as shown in Equation (1a) where costs related to maintenance actions are not included. Moreover, the variable operation cost for the OWF is also considered to be zero (Table 1). Case VI exhibits the lowest average price and operation cost in both deterministic and stochastic studies, which is due to the high level of integration of wind in the operation of the power system. Case I has the second lowest average electricity price and operation cost in both deterministic and stochastic studies, and this is because of the fact that the problem in Case I is a cost-minimization problem, Equation (1).

Table 5. Summary of electricity price and operation cost; deterministic (stochastic).

\begin{tabular}{|c|c|c|}
\hline & Avg.Price (\$/MWh) & Operation Cost (\$) \\
\hline Case I & $23.310(\mathbf{2 3 . 1 1 2})$ & $929,069(923,347)$ \\
\hline Case II & $23.722(23.211)$ & $930,450(924,639)$ \\
\hline Case III & $23.373(23.855)$ & $929,221(924,250)$ \\
\hline Case IV & $23.681(23.755)$ & $930,299(924,687)$ \\
\hline Case V & $23.688(24.090)$ & $930,471(923,995)$ \\
\hline Case VI & $22.948(22.357)$ & $908,732(900,718)$ \\
\hline Case VII & 23.675 (23.892) & $930,222(925,036)$ \\
\hline Case VIII & $23.363(-)$ & $930,855(-)$ \\
\hline Case IX & $25.855(-)$ & $919,536(-)$ \\
\hline
\end{tabular}

Consideration of unexpected outages for the WTs in Case VIII shows that the profit can be rather significantly decreased (Table 4). This is due to the fact that more outages result in less production and hence more loss in profit for the OWF. This is also in line with the increase in the operation cost when compared with Case III (Table 5).

While Case VI considers only one maintenance action for the WTs and generators, Case IX assumes that each of them requires two maintenance actions. This increase in the number of maintenance actions has reduced the profit for the OWF by more than half.

A comparison between the operation costs of three profit-maximization cases of Case II, Case IV and Case V in the deterministic study shows that whenever an anomaly occurs for a WT in the wind farm, the best case from the system's perspective is to re-schedule the maintenance of all WTs (Case IV); otherwise, both the average price and operation cost increase. However, the stochastic study shows that considering the stochasticity can change the outcomes, although the change is not significant. Interestingly, this move (choosing Case IV) is also the best option for the wind farm operator, as then, the operator endures the lowest loss in the profit in the deterministic study (Table 4). This is 
due to the fact that re-scheduling maintenance of all of the WTs will helps the model to obtain the next-best-optimum solution, and this prevents the system from deviating from the optimum operation. It should be reminded here that in this model, the cost of the maintenance action is not considered as part of the operation cost of the system. If only the perspective of the system as a whole is considered, one could also study the impact of an emergency situation through the cost-minimization model presented in Equation (1).

Figure 6 displays the electricity prices in the test system during the study period for Cases II-VII in the deterministic study. The maximum electricity price (58.1 \$/MWh) occurs in Case IV at hour $h=46$ where the available wind power at the offshore farm is $78 \mathrm{MW}$ and the overall demand is $350 \mathrm{MW}$. The reason for this peak price is that at this hour, $G_{3}$ undergoes maintenance, and $G_{5}$, the most expensive generator, enters the system to keep the load balance. It should be reminded that in this case, the maintenance schedules of all power plants (except the OWF) are fixed and considered the same as in Case II for the sake of comparing the impact of a sudden anomaly.

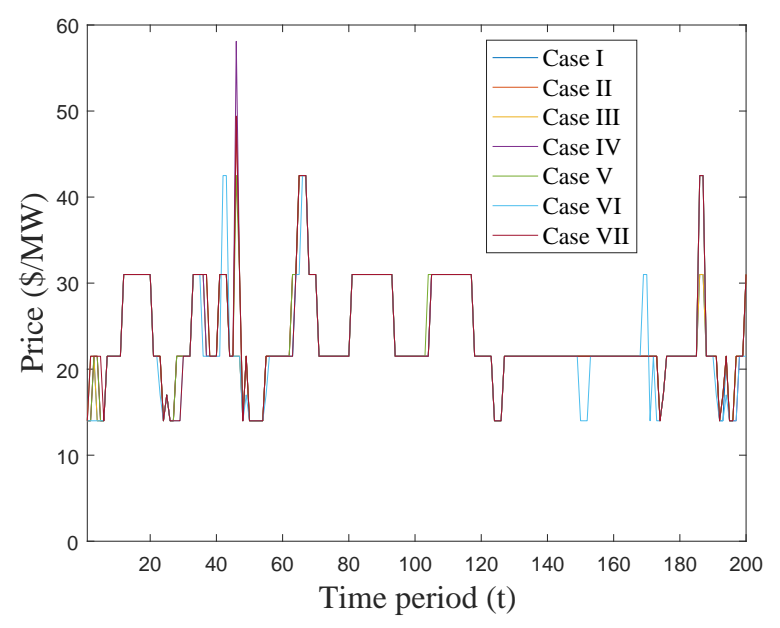

Figure 6. Electricity prices over the study time period for deterministic Cases I-VII.

Although Case VI delivers the lowest electricity average price and operation cost in the deterministic study, few peak values in the prices surge. For instance, during Hours $42-43$ where $G_{2}$ is scheduled for maintenance, the offshore farm does not dispatch its full power, causing the most expensive generator to produce and increase the price. Similar behavior is observed with more prominent impact in Case VI in the stochastic study as the available power in the OWF has increased. On the other hand, the increase in the number of maintenance actions in Case IX has also increased the operation cost in comparison with Case VI, as it has caused more downtime for the OWF. This means more production from conventional generators, which have higher operation costs. Indeed, one can advance this study by introducing the game theory and obtaining the Nash equilibrium in order to evaluate the market power and behavior of other players in the system.

Table 6 shows the frequency of utilization of the vessel types in the Cases I-VII. Please notice that in the currently proposed model, the vessel type does not impact the duration of the maintenance action itself and only affects the transportation time. Therefore, there is no conflict between the vessel optimization and the operation cost; the vessels are only selected based on their cost and optimized based on their availability. Hence, from the arranging vessels point of view, this part only exhibits the applicability of the model with the integration of various vessels and the environmental limitations (wave height). It should also be reminded that there is no difference between the WTs in the same column (Figure 1). The results in both deterministic and stochastic studies show that the system tends to associate the less expensive vessels with the more profitable WTs; in other words, the WTs that are less influenced by the wake effect. As mentioned earlier, the justification for this planning is based on 
the environmental conditions, the costs of the vessels and the time they require for transferring the maintenance crew to and from the offshore farm.

Table 6. Summary of vessel utilization based on WTs in Cases I-VII; deterministic (stochastic).

\begin{tabular}{|c|c|c|c|}
\hline & $b_{1}$ & $b_{2}$ & $b_{3}$ \\
\hline$W T_{1}$ & $3(1)$ & $4(6)$ & $0(\mathbf{0})$ \\
\hline$W T_{2}$ & 0 (1) & $7(6)$ & $0(\mathbf{0})$ \\
\hline$W T_{3}$ & $3(2)$ & $4(4)$ & 0 (1) \\
\hline$W T_{4}$ & $5(2)$ & $2(5)$ & $0(\mathbf{0})$ \\
\hline$W T_{5}$ & 2 (1) & $5(5)$ & 0 (1) \\
\hline$W T_{6}$ & 1 (1) & $6(5)$ & 0 (1) \\
\hline$W T_{7}$ & $3(4)$ & $4(3)$ & $0(\mathbf{0})$ \\
\hline$W T_{8}$ & 0 (1) & $7(6)$ & $0(\mathbf{0})$ \\
\hline$W T_{9}$ & 1 (1) & $6(6)$ & $0(\mathbf{0})$ \\
\hline$W T_{10}$ & 1 (1) & $6(6)$ & $0(\mathbf{0})$ \\
\hline$W T_{11}$ & 1 (1) & $6(6)$ & $0(\mathbf{0})$ \\
\hline$W T_{12}$ & 1 (1) & $6(6)$ & $0(\mathbf{0})$ \\
\hline
\end{tabular}

Regarding the vessel type $b_{3}$, the helicopter, in addition to the mentioned case studies, several other cases were analyzed. For instance, a sensitivity analysis was performed on the cost of the vessels through the deterministic study. In Case I where it is a cost-minimization case, the helicopter was only deployed when its cost became less than twice of the most expensive boat, $b_{2}$. Hence, with low cost, such a case resulted in utilization of vessel $b_{3}$ for the majority of the maintenance of the WTs, and the other WTs used the vessel type $b_{1}$. This also brought about higher profit for the offshore farm and lower operation cost for the system.

Indeed, the most significant value of a helicopter can be considered when boats cannot work due to wave limitations or when there is an urgent need for a maintenance. With this aim, Case IV, amongst the profit-maximization cases, was analyzed where the helicopter was deployed and came into operation with a cost three-times more expensive than the most expensive boat, $b_{2}$. An interesting observation was made as the helicopter was only deployed in the stochastic cases. This shows that the addition of stochasticity could bring about more realistic scenarios where the deterministic study almost disregarded the helicopter completely. One could also further investigate the possibility of studying the impact of postponing a maintenance action in future studies through the proposed SMSOWF model.

\section{Conclusions}

Strategic maintenance scheduling of an offshore wind farm in a deregulated power system is studied in this paper through the proposed SMSOWF model. The model includes practical constraints from the system and the offshore wind farm, such as maintenance working shift, environmental limitation and wake effect. In addition, the SMSOWF model has the capability of integrating condition monitoring information in the operation and planning of the offshore wind farm. The proposed model is assessed from various perspectives through multiple case studies. For instance, the results show that actively considering the market in the operation can bring about significant profit for the offshore wind farm. On the other hand, it is shown that simplifications in terms of neglecting wake effect and working shifts cause the results to deviate from practical outcomes where the wake effect displayed the highest impact.

Acknowledgments: The authors would like to acknowledge the Erasmus Mundus Joint Doctorate (EMJD) Fellowship in Sustainable Energy Technologies and Strategies (SETS) program funded by the European Commission's Directorate-General for Education and Culture, which provided the opportunity for carrying out this work. The authors would also like to thank the anonymous reviewers for their insightful comments and suggestions.

Author Contributions: All authors contributed equally in the development of this paper. 
Conflicts of Interest: The authors declare no conflict of interest.

\section{Nomenclature}

Indices:

$i, j \quad$ Index for bus

$h \quad$ Index for hour

$k \quad$ Index for wind turbine

$g \quad$ Index for generation unit

$s \quad$ Index for stochastic scenario

$r \quad$ Index for maintenance round (number)

Sets:

$\Theta(i) \quad$ Buses connected to bus $i$

$\mathrm{Y}(i) \quad$ Generators connected to bus $i$

Parameters:

$\alpha_{g} \quad$ Incremental term of fuel consumption (MBTU/MW) of generator $g$

$f_{g} \quad$ Cost of fuel (\$/MBTU) of generator $g$

$o_{g} \quad$ Variable operation cost $(\$ / \mathrm{MW})$ of generator $g$

$\bar{Q}_{h, g} \quad$ Maximum capacity (MW) of generator $g \neq O W F$ at time $h$

$\bar{Q}_{h, g, k, s}^{\prime} \quad$ Maximum available wind power (MW) of wind turbine $k$ at time $h$ and scenario $s$ in $g=O W F$

$X_{i, j} \quad$ Inductance $(\Omega)$ of line between buses $i$ and $j$

$F_{i, j} \quad$ Capacity (MW) of line between buses $i$ and $j$

$D_{h, i} \quad$ Demand (MW) at hour $h$ and bus $i$

$T_{k} \quad$ Maintenance action duration for each wind turbine $k$ at OWF

$T_{g}^{\prime \prime} \quad$ Maintenance action duration for $g \neq O W F$

$C \quad$ Maintenance action cost (\$)

Z Maximum simultaneous maintenance actions

$\tau \quad$ Night time parameter

$m_{b} \quad$ Cost factor for vessel $b$

$w_{h, s} \quad$ Wave height prediction $(\mathrm{m})$ at hour $h$ and scenario $s$

$L_{b} \quad$ Wave height limit (m) for vessel $b$

$T_{k, b}^{\prime} \quad$ Transportation time (hour) for vessel $b$ between wind turbine $k$ and shore

$h^{\prime} \quad$ Maximum time limit for maintenance from alarm signal of condition

monitoring system

$k^{\prime} \quad$ Wind turbine that has received an alarm signal

$N_{h} \quad$ Number of time periods

$N_{g} \quad$ Number of generators

$N_{i} \quad$ Number of buses

$N_{S} \quad$ Number of stochastic scenarios

$N_{r} \quad$ Number of maintenance rounds

$M_{1}, M_{2}, M_{3} \quad$ Sufficiently large numbers

Variables:

$q_{h, g, s}$

$\theta_{h, i, s}$

Power generation (MW) of unit $g$ at hour $h$ and scenario $s$

$\begin{array}{ll}z_{h, k, b, r} \quad \text { Binary variable of maint } & \text { maintenance action } r\end{array}$

Voltage angle (rad) in node $i$ at hour $h$ and scenario $s$

$u_{h, k, b, r} \quad$ Binary variable of utilization status of vessel $b$ for WT $k$ at hour $h$ on maintenance action $r$

$y_{h, g, r} \quad$ Binary variable of maintenance status of generator $g$ (except OWF) at hour $h$ on maintenance action $r$ 


$\begin{array}{ll}\mu_{h, i, s} & \text { Electricity price at hour } h \text {, bus } i \text { and scenario } s \\ \lambda_{1_{h, i, s}} & \text { Lagrange multiplier of demand balance } \\ \lambda_{2 h, i, s} & \text { Lagrange multiplier of power-flow } \\ \lambda_{3_{h, g, s}} & \text { Lagrange multiplier of maximum capacity limit for } g \neq O \text { OWF } \\ \lambda_{4_{h, g, b s}} & \text { Lagrange multiplier of maximum capacity limit for } g=O W F \\ \lambda_{5 h, s,} & \text { Lagrange multiplier of capacity positivity } \\ \lambda_{h, g, k, b, s}^{\prime} & \text { Variable used for linearization in strong duality } \\ \lambda_{h, g, s, s}^{\prime \prime} & \text { Variable used for linearization in strong duality }\end{array}$

\section{References}

1. Castro-Santos, L.; Diaz-Casas, V. Resource Assessment Methods in the Offshore Wind Energy Sector. In Floating Offshore Wind Farms; Springer: Basel, Switzerland, 2016; pp. 121-141.

2. Musial, W.; Ram, B. Large-Scale Offshore Wind Power in the United States, Assessment of Opportunities and Barriers; Offshore Wind Hub: Cambridge, MA, USA, 2010; p. 221.

3. Maples, B.; Saur, G.; Hand, M.; van de Pietermen, R.; Obdam, T. Installation, Operation, and Maintenance Strategies to Reduce the Cost of Offshore Wind Energy; Offshore Wind Hub: Cambridge, MA, USA, 2013; p. 89.

4. Tavner, P. Overview of Offshore Wind Development. In Offshore Wind Turbines: Reliability, Availability $\mathcal{E}$ Maintenance; The Institution of Engineering and Technology IET: Stevenage, UK, 2012; p. 296.

5. Van Bussel, G.; Henderson, A.; Morgan, C.; Smith, B.; Barthelmie, R.; Argyriadis, K.; Arena, A.; Niklasson, G.; Peltola, E. State of the Art and Technology Trends for Offshore Wind Energy: Operation and Maintenance Issues. In Proceedings of the Offshore Wind Energy EWEA Special Topic Conference, Brussels, Belgium, 10-12 December 2001.

6. Tavner, P. Maintenance for Offshore Wind Turbines. In Offshore Wind Turbines: Reliability, Availability $\mathcal{E}$ Maintenance; IET: Stevenage, UK, 2012; p. 296.

7. El-Thalji, I.; Liyanage, J.P. On the Operation and Maintenance Practices of Wind Power Asset: A status review and observations. J. Qual. Maint. Eng. 2012, 18, 232-266.

8. Dawid, R.; mcMillan, D.; Revie, M. Review of Markov Models for Maintenance Optimization in the Context of Offshore Wind. In Proceedings of the Annual Conference of the Prognostics and Health Management Society, Coronado, CA, USA, 18-24 October 2015; PHM Society: Coronado, CA, USA, 2015; Volume 6.

9. Abdollahzadeh, H.; Atashgar, K.; Abbasi, M. Multi-Objective Opportunistic Maintenance Optimization of a Wind Farm considering Limited Number of Maintenance Groups. Renew. Energy 2016, 88, 247-261.

10. Sarker, B.R.; Faiz, T.I. Minimizing Maintenance Cost for Offshore Wind Turbines following Multi-level Opportunistic Preventive Strategy. Renew. Energy 2015, 85, 104-113.

11. Shafiee, M.; Finkelsteinb, M.; Berenguer, C. An Opportunistic Condition-based Maintenance Policy for Offshore Wind Turbine Blades Subjected to Degradation and Environmental Shocks. Reliab. Eng. Syst. Saf. 2015, 142, 463-471.

12. Mazidi, P.; Du, M.; Bertling, L.T.; Sanz-Bobi, M.A. A Performance and Maintenance Evaluation Framework for Wind Turbines. In Proceedings of the International Conference on Probabilistic Methods Applied to Power Systems (PMAPS), Beijing, China, 16-20 October 2016.

13. Mazidi, P.; Du, M.; Sanz-Bobi, M.A. A Comparative Study of Techniques Utilized in Analysis of Wind Turbine Data. In Proceedings of the China International Conference on Electricity Distribution (CICED), Xi'an, China, 10-12 August 2016.

14. Mazidi, P.; Bertling, L.T.; Sanz-Bobi, M.A. Wind Turbine Prognostics and Maintenance Management based on a Hybrid Approach of Neural Networks and Proportional Hazards Model. J. Risk Reliab. 2016, doi:10.1177/1748006X16686899.

15. Marugan, A.P.; Marquez, F.P.G.; Perez, J.M.P. Optimal Maintenance Management of Offshore Wind Farms. Energies 2016, 9, 46.

16. Shafiee, M. Maintenance Logistics Organization for Offshore Wind Energy: Current Progress and Future Perspectives. Renew. Energy 2016, 77, 182-193.

17. Dinwoodie, I.; Endrerud, O.E.V.; Hofmann, M.; Martin, R.; Sperstad, I.B. Reference Cases for Verification of Operation and Maintenance Simulation Models for Offshore Wind Farms. Wind Eng. 2015, 39, 1-14. 
18. Hofmann, M. A Review of Decision Support Models for Offshore Wind Farms with an Emphasis on Operation and Maintenance Strategies. Wind Eng. 2011, 35, 1-16.

19. Douard, F.; Domecq, C.; Lair, W. A Probabilistic Approach to Introduce Risk Measurement Indicators to an Offshore Wind Project Evaluation-Improvement to an existing tool. In Proceedings of the 11th International Probabilistic Safety Assessment and Management Conference and the Annual European Safety and Reliability Conference, Helsinki, Finland, 25-29 June 2012; pp. 358-364.

20. Scheu, M.; Matha, D.; Hofmann, M.; Muskulus, M. Maintenance Strategies for Large Offshore Wind Farms. In Proceedings of the Energy Procedia: Deep Sea Offshore Wind R\&D Conference, Trondheim, Norway, 19-20 January 2012; Volume 24, pp. 281-288.

21. Browell, J.; Zitrou, A.; Walls, L.; Bedford, T.; Infield, D. Analysis of Wind and Wave Data to Assess Maintenance Access to Offshore Wind Farms. In Safety, Reliability and Risk Analysis: Beyond the Horizon; CRC Press: Amsterdam, The Netherlands, 2013; pp. 743-750.

22. Besnard, F.; Patrikssont, M.; Strombergt, A.B.; Wojciechowski, A.; Tjernberg, L.B. An Optimization Framework for Opportunistic Maintenance of Offshore Wind Power System. In Proceedings of the 2009 IEEE Bucharest PowerTech, Bucharest, Romania, 28 June-2 July 2009.

23. Besnard, F.; Fischer, K.; Tjernberg, L.B. A Model for the Optimization of the Maintenance Support Organization for Offshore Wind Farms. IEEE Trans. Sustain. Energy 2013, 4, 443-450.

24. Dalgic, Y.; Lazakis, I.; Dinwoodie, I.; McMillan, D.; Revie, M. Advanced Logistics Planning for Offshore Wind Farm Operation and Maintenance Activities. Ocean Eng. 2015, 101, 211-226.

25. Simani, S. Overview of Modelling and Advanced Control Strategies for Wind Turbine Systems. Energies 2015, 8, 13395-13418.

26. Van Bussel, G.; Zaaijer, M. Reliability, Availability and Maintenance Aspects of Large-scale Offshore Wind Farms, a Concepts Study. In Proceedings of the MAREC Marine Renewable Energies Conference, Newcastle, UK, 27-28 March 2001; pp. 119-126.

27. Martin, R.; Lazakis, I.; Barbouchi, S.; Johanning, L. Sensitivity Analysis of Offshore Wind Farm Operation and Maintenance Cost and Availability. Renew. Energy 2016, 85, 1226-1236.

28. Wang, Y.; Handschin, E. A New Genetic Algorithm for Preventive Unit Maintenance Scheduling of Power Systems. Int. J. Electr. Power Energy Syst. 2000, 22, 343-348.

29. Perez-Canto, S.; Rubio-Romero, J.C. A Model for the Preventive Maintenance Scheduling of Power Plants including Wind Farms. Reliab. Eng. Syst. Saf. 2013, 119, 67-75.

30. Mazidi, P.; Bobi, M.A.S. Implementation of Risk in Generation Planning. In Proceedings of the 10th Workshop on Industrial Systems and Energy Technologies, Peradeniya, Sri Lanka, 18-20 December 2015.

31. Froger, A.; Gendreau, M.; Mendoza, J.E.; Pinson, E.; Rousseau, L.M. Maintenance Scheduling in the Electricity Industry: A Literature Review. Eur. J. Oper. Res. 2016, 251, 695-706.

32. Chinneck, J.W. Chapter 20-The Karush-Kuhn-Tucker (KKT) Conditions. In Practical Optimization: A Gentle Introduciton; Carleton University: Ottawa, ON, Canada, 2015.

33. Fortuny-Amat, J.; McCarl, B. A Representation and Economic Interpretation of a Two-Level Programming Problem. J. Oper. Res. Soc. 1981, 32, 783-792.

34. Rosenthal, R.E. GAMS—A User's Guide; GAMS Development Corporation: Washington, DC, USA, 2016.

35. User's Manual for CPLEX; Technical Report; IBM Corp.: Armonk, NY, USA, 2016.

36. National Data Buoy Center, National Weather Service Organization. 2017. Available online: http://www. ndbc.noaa.gov/ (accessed on 1 Febuary 2017).

37. De Andrade Vieira, R.; Sanz-Bobi, M.A. Failure Risk Indicators for a Maintenance Model Based on Observable Life of Industrial Components With an Application to Wind Turbines. IEEE Trans. Reliab. 2013, 62, 569-582.

38. Ribrant, J.; Bertling-Tjernberg, L. Survey of failures in wind power systems with focus on Swedish wind power plants during 1997-2005. In Proceedings of the IEEE Power Engineering Society General Meeting, Tampa, FL, USA, 24-28 June 2007.

39. Report on Wind Turbine Subsystem Reliability - A Survey of Various Databases; Technical Report NREL/PR5000-59111; National Renewable Energy Laboratory NREL: Golden, CO, USA, 2013.

(C) 2017 by the authors. Licensee MDPI, Basel, Switzerland. This article is an open access article distributed under the terms and conditions of the Creative Commons Attribution (CC BY) license (http://creativecommons.org/licenses/by/4.0/). 